\title{
TORCH-a Cherenkov based time-of-flight detector
}

\author{
M.W.U. van Dijk ${ }^{\text {a,*, N.H. Brook }}{ }^{a}$, L. Castillo García ${ }^{\text {b,c }}$, E.N. Cowie ${ }^{a}$, D. Cussans ${ }^{\text {a }}$, \\ C. D'Ambrosio ${ }^{\text {b }}$, J. Fopma ${ }^{\mathrm{d}}$, R. Forty ${ }^{\mathrm{b}}$, C. Frei ${ }^{\mathrm{b}}$, R. Gao ${ }^{\mathrm{d}}$, T. Gys ${ }^{\mathrm{b}}$, N. Harnew ${ }^{\mathrm{d}}$, \\ T. Keri ${ }^{\mathrm{d}}$, D. Piedigrossi ${ }^{\mathrm{b}}$ \\ ${ }^{a}$ H.H. Wills Physics Laboratory, University of Bristol, Tyndall Avenue, Bristol BS8 1TL, United Kingdom \\ ${ }^{\mathrm{b}}$ European Organisation for Nuclear Research (CERN), CH-1211 Geneva 23, Switzerland \\ c Laboratory for High Energy Physics, Ecole Polytechnique Fédérale de Lausanne, CH-1015 Lausanne, Switzerland \\ ${ }^{\mathrm{d}}$ Denys Wilkinson Laboratory, University of Oxford, Keble Road, Oxford OX1 3RH, United Kingdom
}

\section{A R T I C L E I N F O}

\section{Available online 9 May 2014}

Keywords:

Cherenkov radiation

Particle identification

TORCH

MCP-PMT

\begin{abstract}
A B S T R A C T
TORCH is an innovative high-precision time-of-flight system to provide particle identification in the difficult intermediate momentum region up to $10 \mathrm{GeV} / c$. It is also suitable for large-area applications. The detector provides a time-of-flight measurement from the imaging of Cherenkov photons emitted in a $1 \mathrm{~cm}$ thick quartz radiator. The photons propagate by total internal reflection to the edge of the quartz plate and are then focused onto an array of photon detectors at the periphery. A time-of-flight resolution of about 10-15 ps per incident charged particle needs to be achieved to allow a three sigma kaon-pion separation up to $10 \mathrm{GeV} / \mathrm{c}$ momentum for the TORCH located $9.5 \mathrm{~m}$ from the interaction point. Given $\sim 30$ detected photons per incident charged particle, this requires measuring the time-of-arrival of individual photons to about 70 ps. This paper will describe the design of a TORCH prototype involving a number of ground-breaking and challenging techniques.

(c) 2015 CERN for the benefit of the Authors. Published by Elsevier B.V. This is an open access article under the CC BY license (http://creativecommons.org/licenses/by/4.0/).
\end{abstract}

\section{Introduction}

TORCH (Time Of internally Reflected Cherenkov light) has been proposed [1] for the LHCb upgrade to provide charged particle identification (PID) in the low momentum regime (up to $10 \mathrm{GeV} / c$ ), illustrated in Fig. 1. The TORCH detector will supplement the current PID system after removal of the aerogel in RICH-1, which currently performs this task [2]. Optimal kaon-pion separation in the low momentum region is important to LHCb physics for rare $B$ hadronic decays and flavour tagging [2]. It is currently proposed to locate TORCH $9.5 \mathrm{~m}$ from the interaction point, in front of the RICH-2 detector. The LHCb upgrade is scheduled to be installed during the LHC Long Shutdown 2 (LS2) in 2018 although TORCH may come later.

\section{Conceptual design of TORCH}

In TORCH, the time-of-flight (TOF) of a traversing particle is measured by detecting the emitted Cherenkov light. The time of flight difference between kaons and pions of identical momentum

\footnotetext{
* Corresponding author.

E-mail address: m.vandijk@bristol.ac.uk (M.W.U. van Dijk).
}

$p$ over distance $z$ is given by

$t_{K}-t_{\pi}=\frac{z}{c} \frac{1}{2 p^{2}}\left(m_{K}^{2}-m_{\pi}^{2}\right)$.

Here $t_{K}$ and $t_{\pi}$ are the respective times of flight of a kaon (of mass $m_{K}$ ) and a pion (of mass $m_{\pi}$ ). For a $9.5 \mathrm{~m}$ flight path, a per-track time resolution of $10-15$ ps is required for three sigma separation between kaons and pions. To reach this resolution, a DIRC-style

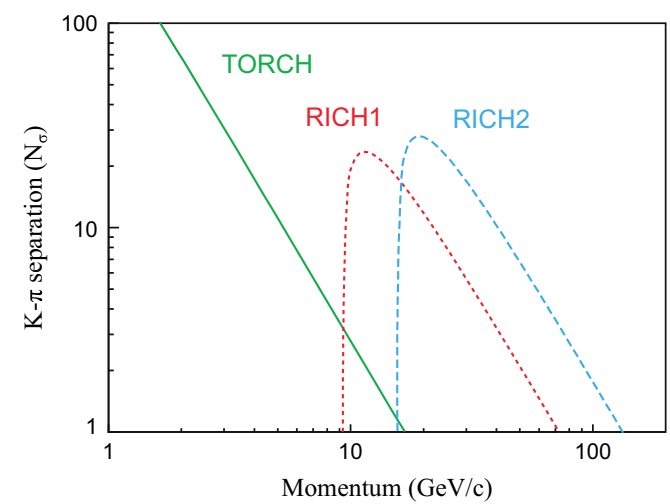

Fig. 1. Calculated performance (in sigma separation) of the different components of the LHCb PID system versus momentum, for isolated tracks [1]. The effect of angular straggling is not included. 

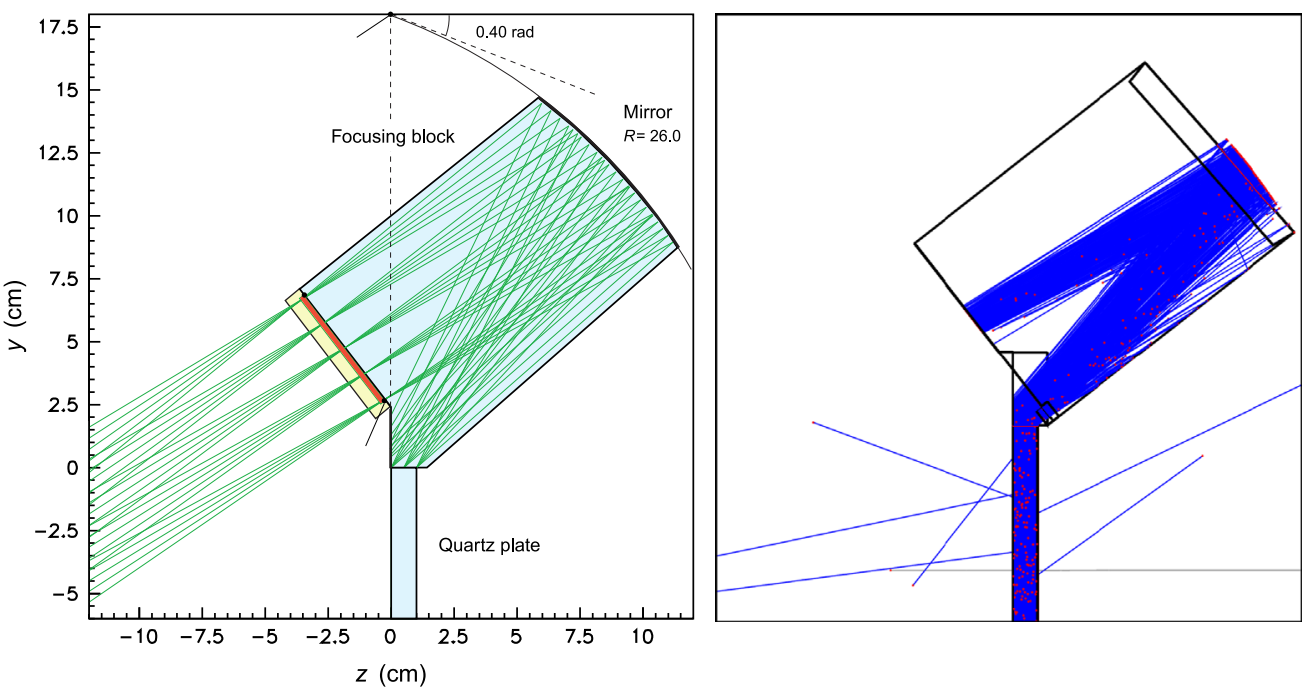

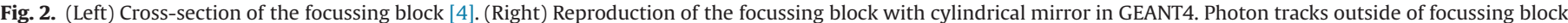
and radiator plate caused by diffuse reflection off rough surface and Rayleigh scattering.

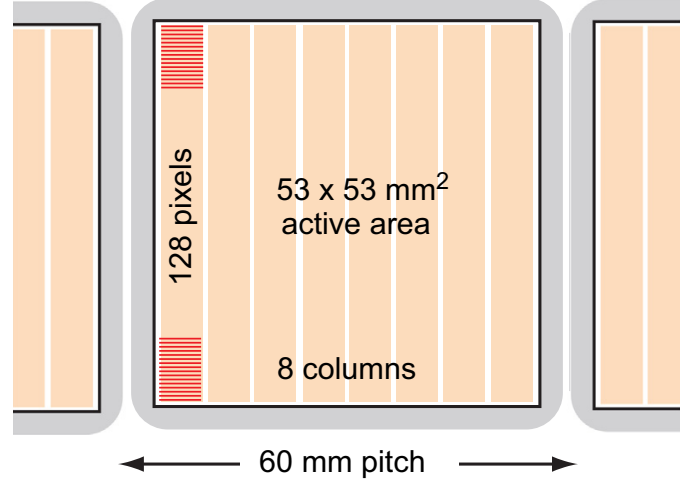

Fig. 3. Schematic of the photon detector layout for TORCH [1].

concept [3] has been proposed for TORCH, using a $1 \mathrm{~cm}$ thick plate of quartz (synthetic fused silica) as a Cherenkov radiator. The photons are propagated to the top and bottom edges of the plate by total internal reflection. A reflecting block then focuses these onto the detector plane.

The number of detected photons directly relates to the intrinsic time resolution of the detector. Given about 30 photons per track [4], and assuming standard $\sqrt{N}$ statistics, this leads to a required intrinsic time resolution for each photon of about $70 \mathrm{ps}$.

The choice of quartz as a medium for both the radiator plate and the focussing block is driven by the high photon yield $(n \approx 1.5)$, its ability to hold a very high polish, and its radiation hardness. These factors are crucial in minimising loss of photons that reach the detector plane. Further factors that require consideration are Rayleigh scattering, the reflectivity of the mirror surface in the focussing block and a spectral cutoff in the UV, typically around $150-200 \mathrm{~nm}$ [3]. The exact requirements on these factors are currently under study using GEANT4 [5] (see Fig. 2). The physical properties used in these studies originate in existing and proposed DIRC detectors, at BaBar [3] and PANDA [6].

The size of the radiator plate is dictated by the angular acceptance of $\mathrm{LHCb}, \pm 0.3 \mathrm{rad}$ in the horizontal plane and $\pm 0.25 \mathrm{rad}$ in the vertical plane. At the proposed downstream location at $9.5 \mathrm{~m}$ this would lead to a rectangular quartz plate of $5 \times 6 \mathrm{~m}^{2}$.

Each detector will be a square $59 \times 59 \mathrm{~mm}$ micro-channel plate photomultiplier tube (MCP-PMT) with an active area of $53 \times$ $53 \mathrm{~mm}^{2}$. The layout of the detector plane is shown in Fig. 3.
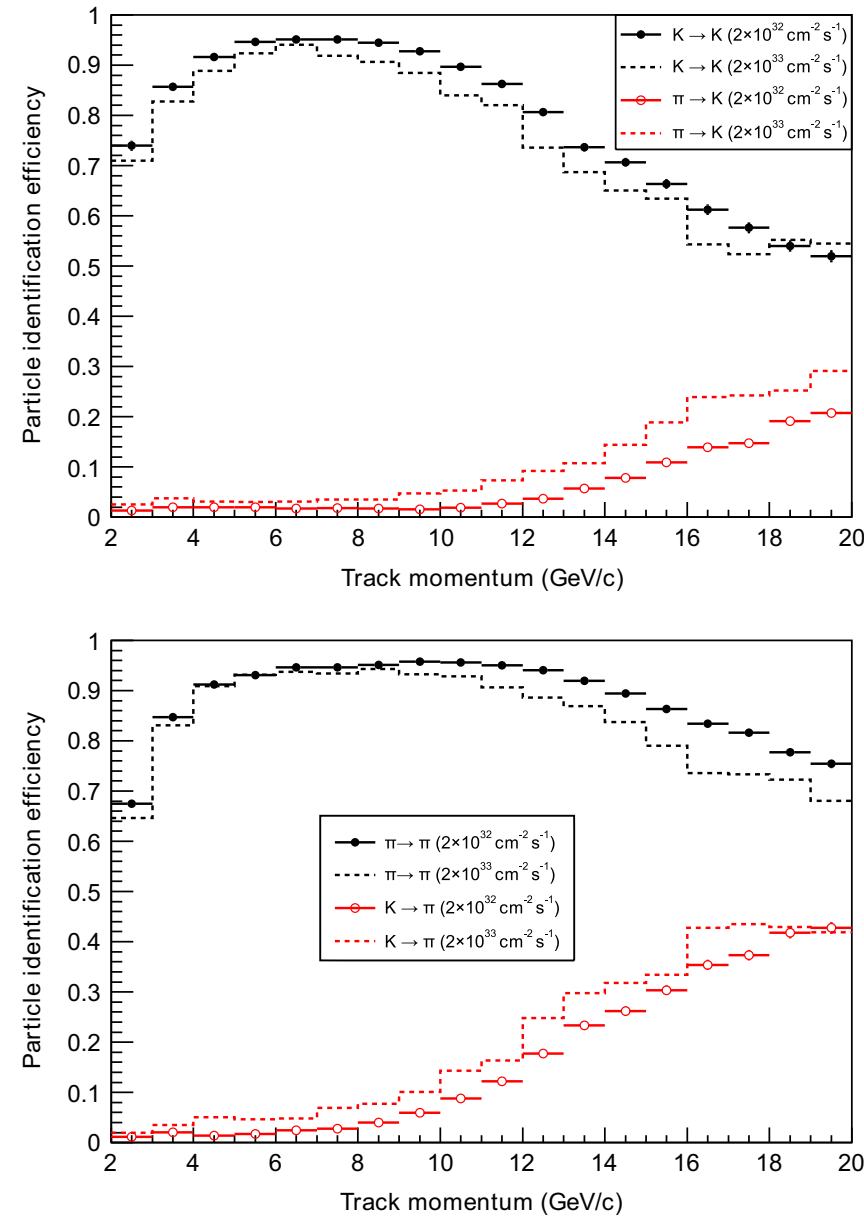

Fig. 4. Particle identification efficiency from the initial design study for the subset of well-measured, truth-matched charged tracks which are matched to a primary vertex. Tracks are assigned as pions or kaons depending on which hypothesis maximises the likelihood, ignoring other mass hypotheses [1]. The top plot shows the efficiency of correctly identifying a kaon (in black) and the chance of mislabelling a pion as a kaon (in red). The bottom plot shows the efficiency of correctly identifying a pion (in black) and the chance of mislabelling a kaon as a pion (in red). These efficiencies are shown for two different luminosities, $2 \times 10^{32} \mathrm{~cm}^{-2} \mathrm{~s}^{-1}$ (points) and $2 \times 10^{33} \mathrm{~cm}^{-2} \mathrm{~s}^{-1}$ (dotted histograms). (For interpretation of the references to color in this figure caption, the reader is referred to the web version of this article.) 


\section{Photons from primary track}

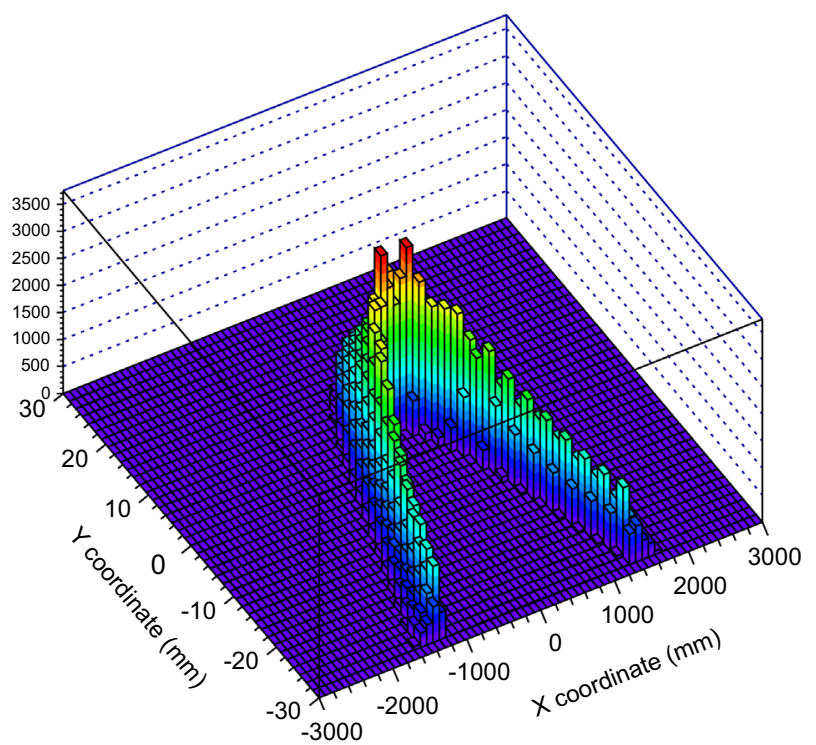

Photons from secondary tracks

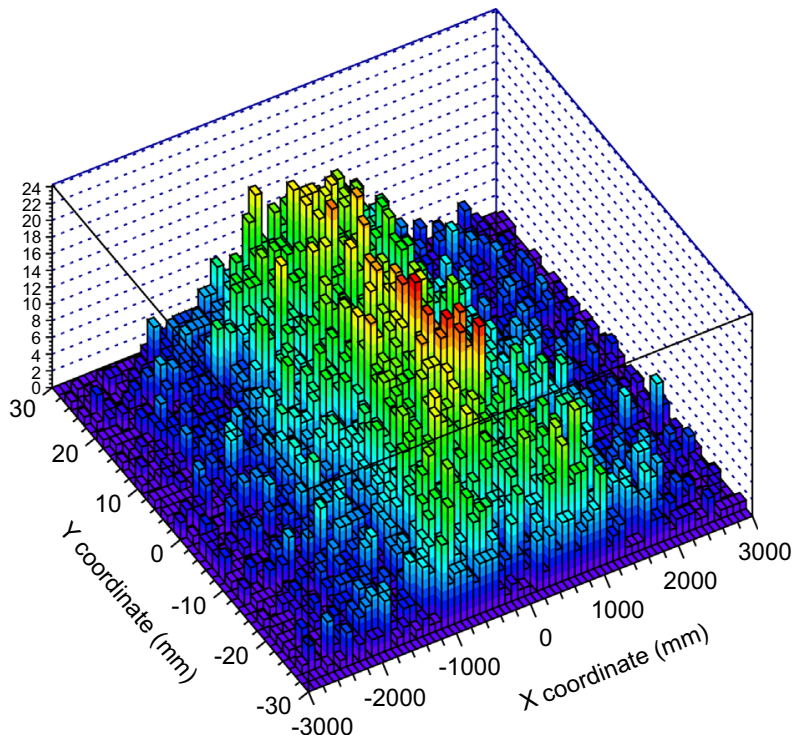

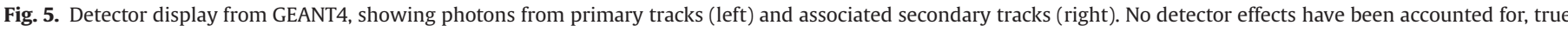

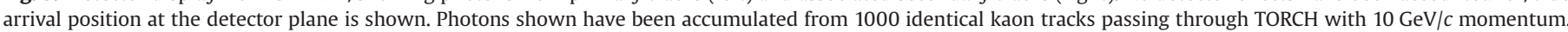
Note that the detector dimensions are highly compressed in one direction due to the detector plane layout.

In order to measure the arrival times of photons that hit the detector plane it is necessary to precisely calculate the path lengths travelled by the photons within the plate and focussing element. To achieve this, information from tracking is used to infer at which point and with which direction the particle traverses the plate. The added effect of angular straggling on the direction of the particle is actively being studied, and will be accounted for in future designs of TORCH.

Due to the focussing block the vertical dimension at the detector plane is a direct measure for the angle of the photon path with the vertical axis $\left(\theta_{z}\right)$. Combining track information with the photon hit position then allows for precise calculation of the path length of the photon. The focussing block has been designed to accept $\theta_{z}$ angles between 0.45 and $0.85 \mathrm{rad}$. This optimises the trade-off between total internal reflection, reflective losses and dispersion. With the detector plane divided vertically into 128 pixels this gives an expected resolution on $\theta_{z}$ of about 1 mrad. In the horizontal dimension, coarse pixelisation of order $6 \mathrm{~mm}$ (8 pixels per detector) is sufficient because there is no focussing and the photons are spread over a greater number of detectors. The error introduced in the calculation of the path length because of pixelisation results in an additional spread in the reconstructed propagation time of the photons. Initial Monte Carlo studies show this effect to be of the order of 55 ps [7].

\section{Time of flight calculation}

Calculating the time of flight of each particle consists of two stages. First, each photon is timed individually. Using the time of propagation through the quartz of photons relative to each other allows for association of the photons with their parent particle. The position where the photon is detected combined with the projected hit position of the parent particle on the plate determines the direction of the photon at production. Combining this with the known trajectory of the parent particle allows the Cherenkov angle of the photon to be calculated. This information can then be used to correct for the effect of chromatic dispersion on the timing of the particle.

To calculate the time of flight, the start time must be calculated. This is done by assuming that particles originating from a single

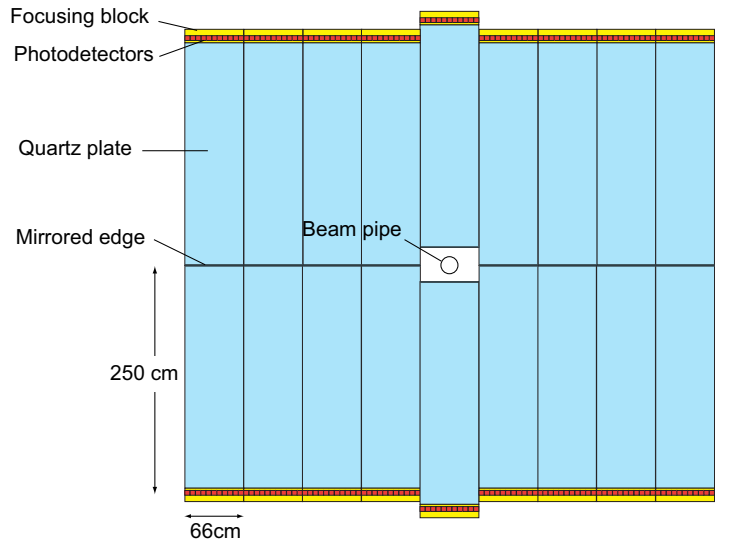

Fig. 6. Schematic layout of the TORCH detector, showing the front view of the 18 identical modules [1].

primary vertex are all pions. The few non-pion tracks give outliers in the histogram of the reconstructed start time $t_{0}$ for a primary vertex and can be rejected. The $t_{0}$ associated with all the particles originating from the same vertex can now be calculated, with an expected time spread of a few picoseconds [7]. The combined reconstructed time information of each single photon associated with a parent particle is combined into the time of arrival of the parent particle at the plate, and together with $t_{0}$ this yields the time of flight from the interaction point.

\section{Photon detectors and electronics}

A customised MCP-PMT that serves the requirements of the TORCH application is currently under development by the Photek company. ${ }^{1}$ This 3 year development project aims to finish around

${ }^{1}$ Photek Ltd., 26 Castleham Road, St. Leonards on Sea, East Sussex, TN38 9NS, United Kingdom. 

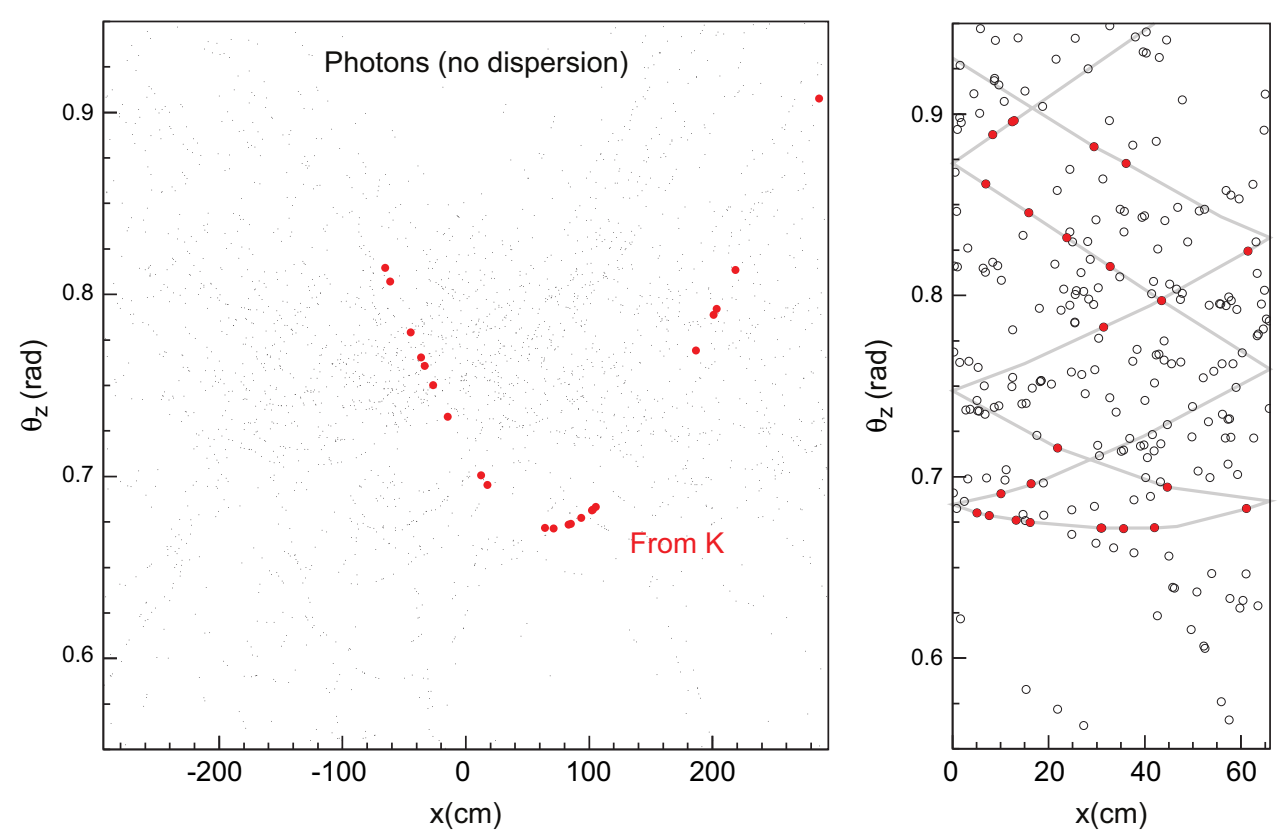

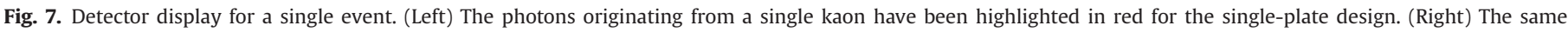

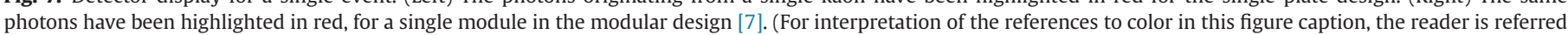
to the web version of this article.)

the end of 2015 [8]. The programme will result in a $59 \times 59 \mathrm{~mm}^{2}$ square MCP with an active area of $53 \times 53 \mathrm{~mm}$. The design is as outlined in Section 2 with $128 \times 8$ pixels, as shown in Fig. 3 .

The expected lifetime of MCPs has been a concern and hence this aspect is an important part of the TORCH MCP-PMT development programme [9]. Recent developments using a special ALD-coating have extended the lifetime of the detectors [10]. Several long-lifetime tubes have been delivered as part of the TORCH programme and are currently undergoing extensive testing. For a single channel device a time resolution of $<30$ ps has been achieved [11].

The front-end electronics system that is planned for TORCH is currently under development [12]. The experimental readout is based on the NINO-8 chipset [13], which provides a time over threshold (TOT) measurement, in conjunction with a high precision time to digital converter (HPTDC) [14]. Using a commercially available $8 \times 8$ channel Planacon MCP (XP85012-A1 ${ }^{2}$ ) with this setup, an overall time resolution of $90 \mathrm{ps}$ is achieved [15]. It is anticipated that TORCH will use a 32-channel version of the NINO. It is expected that further improvements in the readout system will bring the overall single photon time resolution down to the required $70 \mathrm{ps}$.

\section{Simulation}

An initial design study for TORCH using the idealised conceptual design outlined in Section 2 led to the performance estimates shown in Fig. 4. Further development of this study within the GEANT4 framework is currently underway.

The influence of secondaries on the performance of the detector has been studied with GEANT4. Mostly consisting of electrons, the generated secondaries typically have enough energy to be above the Cherenkov threshold. The secondaries subsequently scatter, and the light is mostly uncorrelated with the primary track in the angular direction, but is detected in the same

\footnotetext{
${ }^{2}$ Photonis USA, “XP85012 Planacon Photon Detector”, Rev11-Jan2013. Available: http://www.photonis.com/attachment.php?id_attachment=40.
}

region in the horizontal direction. This is shown in Fig. 5. As such it is expected that the influence of photons from secondaries on the performance of TORCH is negligible.

\section{Modular design of TORCH}

Covering the full area of TORCH $\left(5 \times 6 \mathrm{~m}^{2}\right)$ with a single plate of quartz is not feasible, so a modular design is proposed [1]. This would feature 18 modules of dimensions $66 \times 250 \times 1 \mathrm{~cm}^{3}$, so that a single module covers half the height of the TORCH detector. A conceptual drawing is shown in Fig. 6.

The mirrored edge in the middle of the detector and at the sides of each plate (see Fig. 6) will introduce ambiguities in the reconstruction. The edge of each module at the middle of the radiator plate separates the photons that are going upwards from the photons that are going downward. Reflections on the sides will fold up the curve that would otherwise emerge, as shown in Fig. 7.

One other possible implementation of a modular design that is currently under investigation comes with the possible availability of the quartz used in the former BaBar DIRC. Use of this quartz would circumvent part of the difficult procurement and polishing process at the cost of requiring an optical re-design of the current focussing block.

\section{Conclusion}

The TORCH detector will provide charged particle identification in the $<10 \mathrm{GeV} / c$ momentum range and is proposed as an upgrade to the $\mathrm{LHCb}$ experiment. The programme of R\&D and simulation work will culminate in a prototype TORCH module to demonstrate the feasibility of the full-scale project. Based on initial measurements the aim of $<70$ ps single photon resolution should be attainable. The lifetime issues with MCP detectors are being addressed. Based on simulation work it is expected that photons from secondaries are not an issue. Planning and development for testbeam near the end of 2014 is ongoing. 


\section{Acknowledgments}

The support of the European Research Council is gratefully acknowledged in the funding of this work through an Advanced Grant under the Seventh Framework Programme (FP7), (ERC2011-AdG 299175-TORCH). The authors also thank our industrial partner, Photek Ltd.

\section{References}

[1] The LHCb Collaboration, Letter of Intent for the LHCb Upgrade, CERN-LHCC2011-001, 29 March 2011 (v2).

[2] The LHCb Collaboration, Upgrade TDR: LHCb Particle Identification, CERNLHCC-2013-022, LHCb TDR 14, November 282013.

[3] I. Adam, et al., BaBar Collaboration, Nuclear Instruments and Methods in Physics Research Section A 538 (2005) 281.

[4] M.J. Charles, R. Forty, Nuclear Instruments and Methods in Physics Research Section A 639 (2011) 173.

[5] S. Agostinelli, et al., GEANT4 collaboration, Nuclear Instruments and Methods in Physics Research Section A 506 (2003) 250.
[6] B. Seitz, et al., PANDA collaboration, Nuclear Instruments and Methods in Physics Research Section A 628 (2011) 304.

[7] R. Forty, The TORCH project, Presented at DIRC 2013, Giessen, Germany (4-6 September 2013).

[8] J. Milnes, The TORCH PMT: a close packing, multi-anode, long life MCP-PMT for Cherenkov applications, Presented at DIRC 2013, Giessen, Germany (4-6 September 2013).

[9] J. Milnes, The TORCH PMT, a close packing, multi-anode, long life MCP-PMT for Cherenkov applications, Presented at RICH 2013, Shonan Village, Japan (2-6 December 2013).

[10] T.M. Conneely, J.S. Milnes, J. Howorth, Nuclear Instruments and Methods in Physics Research Section A 732 (2013) 388.

[11] T. Gys, Performance and lifetime of micro-channel plate tubes for the TORCH detector, Presented at RICH 2013, Shonan Village, Japan (2-6 December 2013)

[12] R. Gao, Development of precision time-of-flight electronics for $\mathrm{LHCb} \mathrm{TORCH}$, Presented at TWEPP 2013, Perugia, Italy (23-27 September 2013).

[13] F. Anghinolfi, P. Jarron, A.N. Martemiyanov, et al., Nuclear Instruments and Methods in Physics Research Section A 533 (2004) 183.

[14] M. Mota, J. Christiansen, S. Débieux, et al., IEEE Nuclear Science Symposium Conference Record 2 (2000) 9/155.

15] L. Castillo García, Timing performance of a MCP photon detector read out with multi-channel electronics for the TORCH system, Presented at 14th ICATPP Conference, Villa Olmo, Italy (23-27 September 2013). 